\title{
The association of low voltage atrial electrograms and the myocardial substrate for atrial fibrillation
}

\begin{abstract}
Abnormal human atrial muscle has elevated resting membrane potential, depressed maximal amplitude of the action potential, and decreased upstroke velocity. These electrical abnormalities are histologically correlated to degenerative changes of the atrial muscle structure. Investigations based on the recording of abnormally prolonged and fractionated atrial endocardial electrograms during sinus rhythm have provided important knowledge about the electrophysiological properties of the pathological atrium. Human atrial tissues, where fractionated electrograms are recorded, consist of muscle fibers that are widely separated and their orientation distorted by connective tissue. These histological changes results in decreased intercellular connections, which might increase resistance to current flow, and thereby slow conduction. The potential clinical significance is that detection of abnormal atrial endocardial electrograms during sinus rhythm in patients with atrial vulnerability may help to identify those with greater atrial conduction defects and higher incidence of induced and spontaneous episodes of AF. 3D electroanatomic mapping (EAM) has been utilized in clinical electrophysiological studies for substrate description in atrial arrhythmias. Using this 3D EAM system low bipolar endocardial voltage can be identified and localized for guiding catheter ablation. Voltage mapping with 3D electroanatomic mapping is based on the correlation of the registration of low-voltage areas $(<0.5 \mathrm{mV})$ in the left atrium with endocardial scar and/or structural defects as an atrial substrate. These low-voltage zones are the target for individualized substrate ablation or modification. Patients with low-voltage substrate have lower success rates after pulmonary vein ablation. However, this low success rate can be significantly improved by targeting atrial substrate zones with low voltage areas. Although more clinical evidence should be further generated, patient specific substrate ablation targeting atrial fibrosis estimated by bipolar voltage mapping is a promising alternative strategy for additional substrate modification beyond PVI ablation for $\mathrm{AF}^{1-6}$
\end{abstract}

Keywords: electroanatomic mapping, PVI ablation, atrial muscle, atrial fibrillation, $3 \mathrm{D}$,abnormal atrial electrograms
Volume 12 Issue 6 - 2019

\author{
Osmar Antonio Centurión, ${ }^{1,2}$ José $\mathrm{F}$ \\ Alderete,' José C Candia,' Laura B García, 1,2 \\ Karina E Scavenius, ${ }^{1,2}$ Judith MTorales, ${ }^{1,2}$ Luis \\ M Miñol,2 \\ 'Division of Cardiovascular Medicine, Clinic Hospítal, Asuncion \\ National University (UNA), Paraguay \\ ${ }^{2}$ Department of Health and Sciences Investigation, Sanatorio \\ Metropolitano, Paraguay
}

Correspondence: Osmar Antonio Centurión, Professor of Medicine, School of Medical Sciences, Asunción National University (UNA), Paraguay, Tel +(595) 97I 354444,

Email osmarcenturion@hotmail.com

Received: August 08, 2019 | Published: December 16, 2019
Abbreviations: EAM, electroanatomic mapping; AF, atrial fibrillation; AAE, abnormal atrial electrograms; LVZ, low voltage zone

\section{Introduction}

Atrial fibrillation (AF) is a common arrhythmia associated to lethal complications such as clinical outcomes. Pathological changes in the atrial myocardium may lead to electrophysiological produce decreasing local voltage, conduction block and low electrogram amplitude. Indeed, atrial degenerative changes of the atrial muscle structure. $^{7}$

Investigations based on the recording of abnormally prolonged and fractionated atrial local electrograms during sinus rhythm have provided important knowledge about the electrophysiological properties of the pathological atrium. ${ }^{8-12}$ Human atrial myocardium where abnormal atrial electrograms are recorded, consist of muscle fibers that are widely separated and their orientation distorted by collagen. These histological changes result in decreased intercellular connections, which might increase resistance to current flow, and thereby slow conduction. ${ }^{13-15}$ Therefore, abnormally prolonged and fractionated atrial electrograms result in an irregular atrial conduction. This irregularity is characterized by a non-homogeneous local electrical activity, related to an anisotropic, non-uniform and delayed conduction through a pathological atrial myocardium. ${ }^{16-18}$ In such altered atrial substrate arrhythmias may develop due to reentry. The 3D electroanatomic mapping (EAM) revealed anatomic and structural atrial abnormalities that were associated with a change in the nature of sinus pacemaker activity. In addition, there were loss of the normal multi-centric pattern of onset of sinus impulse, shift of the pacemaker complex to low crista terminalis sites, and the presence of abnormal and circuitous conduction around lines of conduction block. 3D electroanatomic bipolar voltage mapping is a diagnostic auxiliary method that has been utilized in clinical electrophysiological studies for substrate description in atrial arrhythmias. ${ }^{19-21}$ Recent advances in 3D EAM systems have facilitated catheter manipulation within the atrium at reduced fluoroscopy exposure time. Using this 3D EAM system low bipolar endocardial voltage can be identified and localized for guiding catheter ablation. The electrical information obtained can be used in different ways to record different information. For example, certain map points recorded can be utilized for the color-coded display of the electrical activation sequence known as "activation mapping". In addition, the display of post-pacing intervals known as "entrainment mapping" can be recorded, as well as, the 
display of unipolar/bipolar electrograms as part of "fractionation" or "voltage mapping". ${ }^{19-21}$ Therefore, we aim to analyze the association of low voltage atrial electrograms and the myocardial substrate for the development of atrial fibrillation.

\section{Abnormal atrial electrograms and the myocardial substrate}

During an atrial depolarization the axial current flows from one myocardial cell to the adjacent one through the intercalated discs, which normally have a relatively low resistance..$^{22}$ Under normal tissue conditions some mild disturbances in conduction of cardiac impulses may occur. The conduction perpendicular to the longitudinal axis of the atrial muscle fibers can be delayed because in that direction the effective axial resistance is greater. ${ }^{22}$ This greater axial strength results in part from the scarce amount and short length of the intercalated discs in the lateral-lateral direction of the muscle fibers, compared to the terminal-terminal direction. There is an added alteration to the electrical conduction when the atrial muscle fibers are separated by connective tissue. In this context, the conduction properties can be further altered due to the effects on the axial resistance creating a discontinuous and anisotropic propagation. ${ }^{22-24}$ In this regard, Spach et al., ${ }^{24}$ have demonstrated in a nicely done work that the slowing of the conduction velocity causes a decrease in the amplitude and, an increase in the duration of the extracellular electrogram of the canine Purkinje system. In a computerized model of electrogram generation study, Lesch et al., ${ }^{25}$ has shown that the decreased conduction velocity was responsible for the increase in electrogram duration, while increased intracellular resistance was responsible for the fractionation of the electrogram.

More than a quarter of century ago, Centurión et al., ${ }^{9}$ designed a study to assess the importance and significance of recording of abnormal atrial electrograms (AAE) during sinus rhythm in $\mathrm{AF}$ patients (Figure 1). We showed that patients who had AAE had a significantly increased atrial vulnerability compared to those with normal electrograms. Abnormal atrial electrograms showed a very good specificity and positive predictive value when evaluating the induction of sustained AF. The specificity demonstrated was $94 \%$ with a positive predictive value of $93 \% .{ }^{9}$ We have also demonstrated a significantly longer conduction time of sinus impulses in AF patients who possess AAE within the right atrium. In our study the mean intra-atrial $(54 \mathrm{~ms})$ and the mean interatrial conduction time $(100 \mathrm{~ms})$ of sinus impulses were significantly longer in the AAE (+) Group. ${ }^{9}$ In addition, AAE also proved to be a useful marker to differentiate patients with more extensive atrial electrical abnormalities who are more likely to have inducible AF. Prolonged and fractionated AAE may reflect inhomogeneous local electrical activity related to a delayed and non-uniform anisotropic conduction through a diseased atrial muscle (Figure 2). The potential clinical significance is that detection of AAE during sinus rhythm in patients with atrial vulnerability may help to identify those with greater atrial conduction defects and higher incidence of induced and spontaneous episodes of AF.
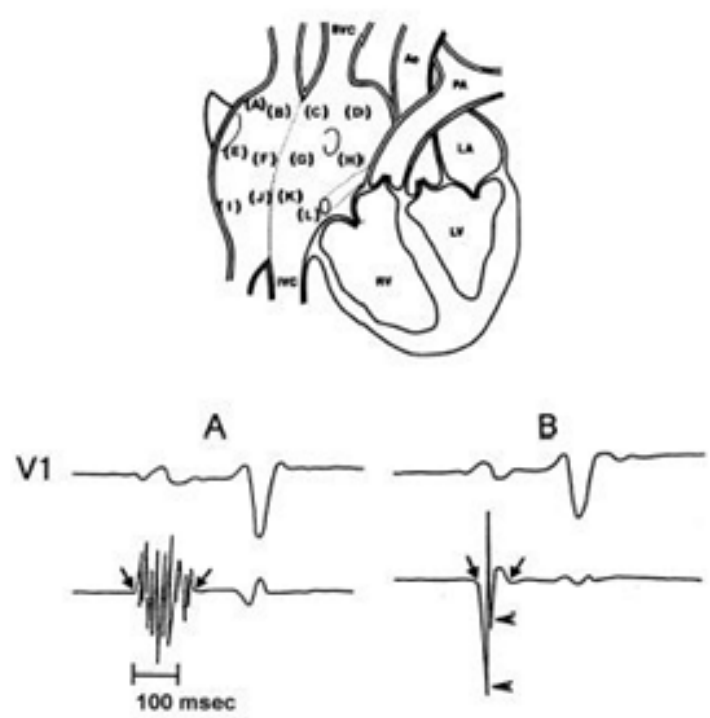

Figure I Sites of endocardial catheter mapping of the right atrium are depicted.

The atrial electrograms were recorded from 12 endocardial sites in each patient. The sites listed from $A$ to $D$ correspond to the upper part of the right atrium while the sites from $E$ to $H$ are from the middle part, and those from I to $L$ are from the lower part of the right atrium. The sites marked $A$, $E$ and I correspond to the anterior region, sites $B, F$ and $J$ to the lateral region, sites $C, G$ and $K$ to the posterior region, and sites $D, H$ and $L$ correspond to the medial region of the right atrium.

SVC: superior vena cava. IVC: inferior vena cava.Ao:Aorta. PA: pulmonary artery. LA: left atrium. RV: right ventricle. LV: left ventricle.

In the lower part (A) there is an abnormal endocardial atrial electrogram with a duration of 130 ms and 10 fragmented deflections. (B) shows a normal endocardial atrial electrogram that lasts $80 \mathrm{~ms}$ in duration and has two fragmented deflections. Reprinted with permission from Centurion OA et al. Influence of advancing age on fractionated right atrial endocardic electrograms. Am J Cardiol 2005; 96: 239-242. 
A

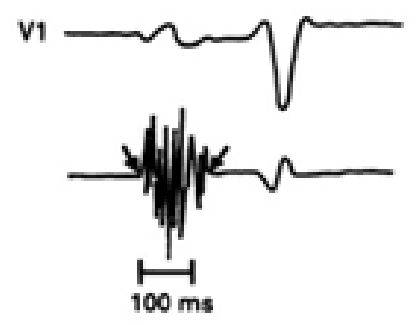

D

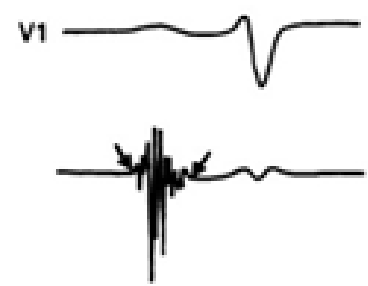

B

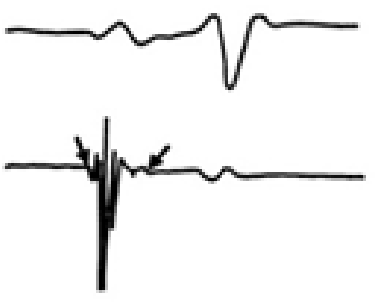

E
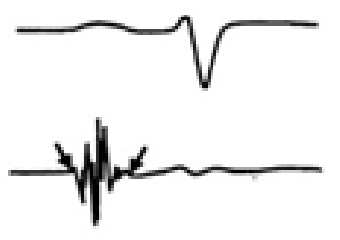

C
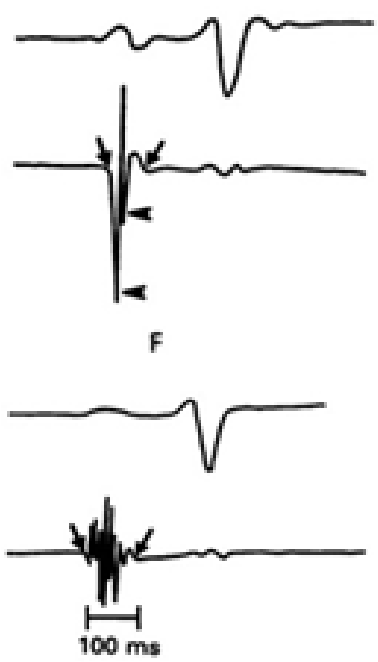

Figure 2 Six atrial electrograms recorded at different right atrial sites in two patients with Sinus Node Dysfunction (A, B, and C in a SND patient with Rubenstein Type I and II; D, E, and F in a SND patient with Rubenstein Type III). Diagonal arrows represent the onset and offset of local electrical activity and horizontal arrows the downward deflections. Reprinted with permission from Centurión OA, Fukatani M, Konoe A, Tanigawa M, Shimizu A, Isomoto $S$ y col. Different distribution of abnormal endocardial electrogram within the right atrium in patients with sick sinus syndrome. Br Heart J 1992;68:596-600.

\section{Atrial low voltage zones in 3D electroanatomic mapping}

3D electroanatomic mapping (EAM) has been utilized in clinical electrophysiological studies for substrate description in atrial arrhythmias. Using this 3D EAM system low bipolar endocardial voltage can be identified and localized for guiding catheter ablation. Voltage mapping with $3 \mathrm{D}$ electroanatomic mapping is based on the correlation of the registration of low-voltage areas $(<0.5 \mathrm{mV})$ in the left atrium with endocardial scar and/or structural defects as an atrial substrate (Figure 3). ${ }^{26-29}$ These low-voltage zones are the target for individualized substrate ablation or modification. Patients with lowvoltage substrate have lower success rates after pulmonary vein ablation. However, this low success rate can be significantly improved by targeting atrial substrate zones with low voltage areas. In a study with 3D electroanatomic mapping, it was found an age- related evidence of generalized conduction slowing in the atria. ${ }^{30-33}$ They also demonstrated increasing numbers of fractionated electrograms and double potentials in the atrium but along the crista terminalis with aging in patients prone to develop $\mathrm{AF}^{32}$ This fact may be important because functional conduction delay at this structure has been implicated in the development of a range of atrial arrhythmias. In this regard, Sanders et al., ${ }^{33}$ designed a study with 3D electroanatomic mapping in order to search for evidence of diffuse atrial abnormalities in patients with a propensity to develop AF. They performed 3D EA mapping to assess regional conduction velocity, double potentials, fractionated electrograms, regional voltage, and areas of scar tissue. They demonstrated significant increase in atrial ERP at all right atrial sites, increased atrial conduction time along the lateral RA and $\mathrm{CS}$, prolongation of the P-wave duration, and greater number and duration of double potentials along the crista terminalis. ${ }^{33}$ The authors described a significant regional conduction slowing with double potentials and fractionation associated with areas of low voltage and scar. These regions of low voltage and fibrous tissue were particularly marked along the crista terminalis. These abnormalities resulted in propagation detours and colliding activation wave fronts of atrial activation..$^{33}$

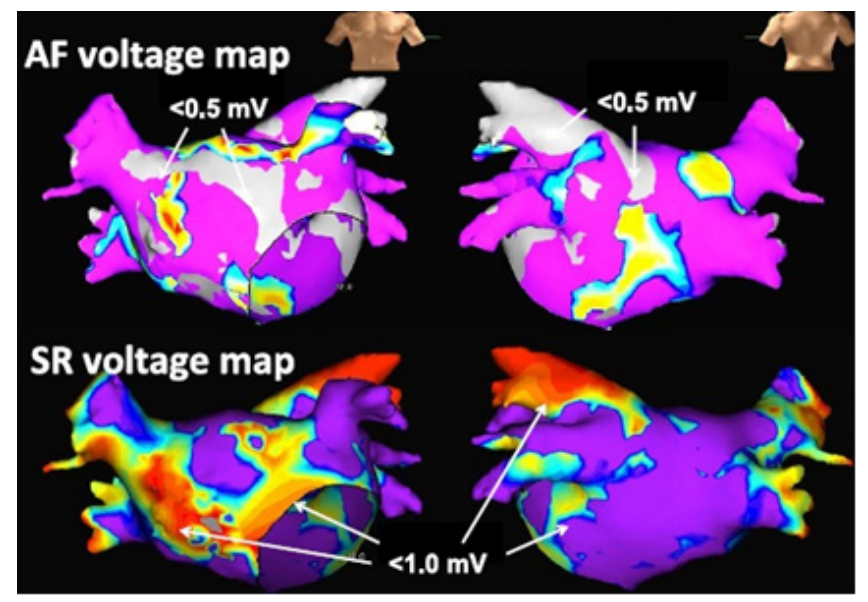

Figure 3 Mapping images of areas of left atrial bipolar voltage during atrial fibrillation (AF, upper panel) and sinus rhythm (SR, lower panel).

Low-voltage areas during AF were defined as those of less than $0.5 \mathrm{mV}$ and are shown in grey color. Low-voltage areas during SR were defined as those of less than $1.0 \mathrm{mV}$ and are shown in non-purple colors. Reprinted with permission from Sasaki N,Watanabe I, Okumura Y, Nagashima K, Kogawa R, Sonoda K, et al. Complex fractionated atrial electrograms, high dominant frequency regions, and left atrial voltages during sinus rhythm and atrial fibrillation. J Arrhythmia 2017;33:185-191.

Roberts-Thomson et al., ${ }^{34}$ observed that fractionated atrial electrograms increase in prevalence with age and occur predominantly along the high posterior and high septal right atrium. However, Bao et al., ${ }^{35}$ found that patient's age was similar among groups, suggesting that the findings cannot be explained by the aging process alone. 
Indeed, their patients with prolonged sinus pauses had a higher percentage of complex electrograms, higher right atrium volumes, and lower voltage in the high lateral right atrium than control patients regardless of age. In previous studies it was found remodeling changes of both atria in lone-AF patients, including structural abnormalities, lower conduction velocities, increased effective refractory periods, abnormal atrial histologic results, and inflammation in patients with sinus pauses greater than 3 seconds or unexplained sinus bradycardia $\left(<40\right.$ beats/min) ${ }^{36-38}$

It is well known that $\mathrm{AF}$ is associated with structural, electrical, and contractile remodeling of the atria. Atrial fibrosis certifies structural remodeling of the atrium and is considered to be the substrate for AF maintenance. ${ }^{39-43}$ Atrial fibrosis yields the pathological substrate to promote $\mathrm{AF}$ by interfering depolarization wave continuity through the atrial myocardium, developing conduction alterations, and causing anisotropic tissue conduction. ${ }^{42}$ Human histopathological results demonstrated that large extent of atrial fibrosis is found in the atria of AF patients and, the extension of atrial fibrosis was significantly associated with AF and duration of the arrhythmia. ${ }^{44-48}$ Atrial septal biopsies obtained from patients with lone paroxysmal AF have revealed inflammatory infiltrates consistent with myocarditis and fibrosis. ${ }^{45}$

Low-voltage electrograms are produced by diverse mechanisms and, the primary mechanism has been suggested to be atrial fibrosis. ${ }^{49-52}$ These atrial changes may reflect poor fiber coupling, non-homogeneous conduction, and anisotropic tissue depolarization caused by atrial myocardial fibrosis. The definition of low voltage zone (LVZ) still remains controversial, mainly due to lack of histopathological studies. Sanders et al., 32,33 tried to define areas of low voltage. They performed electroanatomical maps of the right atrium by pacing the distal coronary sinus or mapping during sinus rhythm in order to study the electrophysiological atrial substrate for AF patients. They identified electrically silent areas, namely, scar defined by the absence of recordable activity or a bipolar voltage amplitude $\leq 0.05$ $\mathrm{mV}$; and low-voltage areas defined by atrial zones with bipolar voltage $\leq 0.5 \mathrm{mV}$. Later on, Verma et al., ${ }^{28}$ utilized the same definition criteria for the left atrium. They demonstrated that pre-existent left atrial fibrosis is a significant independent predictor of recurrence after PVI. Yamaguchi et al., ${ }^{29}$ contrasted the long-term clinical outcomes after PVI alone in patients with and without LVZs defined as $<0.5 \mathrm{mV}$. The incidence of AF free- survival after PVI alone was significantly lower in patients with LVZs, and the extent of the LVZ were identified to be an independent risk factor. Masuda et al.,$^{53}$ also demonstrated similar results showing that the presence of LVZ predicts AF recurrence. The DECAAF multicenter and prospective clinical trial showed that atrial fibrosis estimated by LGE-MRI was independently associated with the likelihood of $\mathrm{AF}$ recurrence in patients undergoing $\mathrm{AF}$ ablation. ${ }^{26}$

It is undeniable the useful help of delayed-enhancement MRI in detecting atrial fibrosis. The group of Marrouche et al., ${ }^{54}$ reported on the efficacy and utility of LGE-MRI in identifying areas of fibrosis in the left atrium, and observed that an enlarged size of left atrial wall enhancement was significantly related to recurrence after PVI ablation. They also found a significant correlation between enhancement on LGE-MRI and low-voltage areas on voltage-guided mapping. It is very interesting that left atrial wall biopsies showing fibrosis were associated with zones of left atrial wall enhancement on LGE-MRI On the contrary, areas with normal biopsy tissue were correlated with non-enhanced regions, demonstrating how accurate LGE-MRI is for atrial fibrosis detection.
There are several studies showing that $\mathrm{AF}$ ablation targeting atrial fibrosis by voltage mapping may improve clinical results. Indeed, several investigations demonstrated the effectiveness of voltage mapping of the atrium in persistent AF patients. ${ }^{55-64}$ However; the limitation is that most of these investigations were small and observational studies in nature. A couple of years ago, Yang et al. ${ }^{65}$ demonstrated similar success results in the one year and a half follow-up, with lower procedure time and fluoroscopy exposition and durations, as well as, shorter energy delivery time. It is very interesting the fact that over half of non-paroxysmal $\mathrm{AF}$ patients did not need further ablation beyond pulmonary vein isolation. ${ }^{55-59}$ Last year, Kircher et al. ${ }^{66}$ also observed the effectiveness of AF ablation guided by voltage mapping in a randomized single-center research. The START Trial is an ongoing large, multicenter, randomized study that was designed to analyze the safety and efficacy of left atrial voltage-based ablation comparing two AF ablation strategies, namely, PVI plus LVZ homogenization Vs. PVI alone in patients with LVZ (https://www.umin. ac.jp/ctr/index.htm, UMIN000022119). This ongoing trial will provide further knowledge into this AF ablation strategy. ${ }^{67}$

\section{Conclusion}

In conclusion, investigations based on conventional atrial catheter mapping with the recording of abnormally prolonged and fractionated atrial local electrograms during sinus rhythm, as well as, their characteristic distribution within the right atrium have provided important knowledge about the electrophysiological properties of the pathological atrial myocardium in AF patients. The detection of abnormal atrial electrograms during sinus rhythm identifies a group of patients with increased atrial vulnerability and a significantly higher incidence of spontaneous or induced episodes of atrial fibrillation. The 3D EAM revealed anatomic and structural atrial abnormalities and the presence of abnormal and circuitous conduction around lines of conduction block. Although more clinical evidence should be further generated, patient specific substrate ablation targeting atrial fibrosis with low voltage zones estimated by bipolar voltage mapping is a promising alternative strategy for additional substrate modification beyond PVI ablation for AF.

\section{Acknowledgements}

None.

\section{Conflicts of interests}

Author declares that there is no conflict of interest.

\section{References}

1. Bourassa MG, Gurne O, Bangdiwala SI, et al. Natural history and patterns of current practice in heart failure. J Am Coll Cardiol. 1993;22(4 Suppl A):14A-19A.

2. Mathew J, Hunsberger S, Fleg J, et al. Incidence, predictive factors, and prognostic significance of supraventricular tachyarrhythmias in congestive heart failure. Chest. 2000;118(4):914-922.

3. Tanigawa M, Fukatani M, Konoe A, et al. Prolonged and fractioned right atrial electrograms during sinus rhythm in patients with paroxysmal atrial fibrillation and sick sinus node syndrome. J Am Coll Cardiol. 1991;17(2):403-408.

4. Hordof AJ, Edie R, Malm JR, et al. Electrophysiologic properties and response to pharmacologic agents of fibers from diseased human atria. Circulation. 1976:54(5):774-779. 
5. Hordof A, Spotnitz A, Mary-Rabine L, et al. The cellular electrophysiologic effects of digitalis on human atrial fibers. Circulation. 1978;57(2):223-229.

6. Gelband H. Bush H, Rosen MR, et al. Electrophysiologic properties of isolated preparations of human atrial myocardium. Circ Res. 1972:30(3):293-300.

7. Mary-Rabine L, Albert A. Hordof A, et al. The relationship of human atrial cellular electropbysiology to clinical function and uitrastrnctnre. Circ Res. 1983:52:188-199.

8. Centurión OA, Fukatani M, Konoe A, et al. Different distribution of abnormal endocardial electrogram within the right atrium in patients with sick sinus syndrome. Br Heart J. 1992;68(6):596-600.

9. Centurion OA, Isomoto S, Fukatani M, et al. Relationship between atrial conduction defects and fractionated atrial endocardial electrograms in patients with sick sinus syndrome. Pacing Clin Electrophysiol. 1993;16(10):2022-2033.

10. Centurion OA, Fukatani M, Konoe A, et al. Electrophysiological abnormalities of the atrial muscle in patients with sinus node dysfunction without tachyarrhythmias. Int J Cardiol. 1992;37(1):41-50.

11. Centurión OA, Shimizu A, Isomoto S, et al. Influence of advancing age on fractionated right atrial endocardial electrograms. Am J Cardiol. 2005;96(2):239-242.

12. Centurión OA, Isomoto S, Shimizu A, et al. The effects of aging on atrial endocardial electrograms in patients with paroxysmal atrial fibrillation. Clin Cardiol. 2003;26(9):435-438.

13. Spach MS, Dolber PC. Relating extracellular potentials and their derivatives to anisotropic propagation at microscopic level in human cardiac muscle: Evidence for electrical uncoupling of side-to-side fiber connections with increasing age. Circ Res. 1986;58(3):356-371.

14. Spach MS, Dolher PC. Relating extracellular potentials and their derivatives to anisotropic propagation at a microscopic level in human cardiac muscle. Circ Res. 1986; 58(3):356-371.

15. Spach MS, Miller WT, Dolber PC, et al. The functional role of structural complexities in the propagation of depolarization in the atrium of the dog. Cardiac conduction disturbances due to discontinuities of effective axial resistivity. Circ Res. 1982;50(2):175-191.

16. Centurion OA, Isomoto S, Shimizu A. Electrophysiological changes of the atrium in patients with lone paroxysmal atrial fibrillation. J Atr Fibrillation. 2010;3(1):232.

17. Centurión OA. Age-related electrophysiological changes of the atrial myocardium in patients with paroxysmal atrial fibrillation. $J$ Cardiol Curr Res. 2015;3(6):00121.

18. Centurión OA, Alderete JF, Torales JM, et al. Electrophysiological Abnormalities of the Atrial Myocardium and the Rubenstein Clinical Classification of Patients with Sinus Node Dysfunction. EC Cardiology. 2018;5(11):846-857.

19. Kumagai K, Nakano M, Kutsuzawa D, et al. The efficacy of ablation based on the combined use of the dominant frequency and complex fractionated atrial electrograms for non- paroxysmal atrial fibrillation. J Cardiol. 2016;67(6):540-50.

20. Knackstedt C, Schauerte P, Kirchhof P. Electro-anatomic mapping systems in arrhythmias. Europace. 2008;10(Suppl 3):28-34.

21. Kimura M, Sasaki S, Owada S, et al. Validation of accuracy of threedimensional left atrial CartoSound and CT image integration: influence of respiratory phase and cardiac cycle. J Cardiovasc Electrophysiol. 2013;24(9):1002-1007.

22. Spach MS, Miller WT 3rd, Geselowitz DB, et al. The discontinuous nature of propagation in normal canine cardiac muscle: Evidence for recurrent discontinuities of intracellular resistance that affect the membrane currents. Circulation Res. 1981;48(1):39-54.

23. Spach MS, Kootsey JM. The nature of electrical propagation in cardiac muscle. Am J Physiol-Heart Circ Physiol. 1983;244(1):H3-H22.

24. Spach MS, Dolber PC, Heidlage JF. Interaction of inhomogeneities of repolarization with anisotropic propagation in dog atria: A mechanism for both preventing and initiating reentry. Circulation Res. 1989;65(6):1612-1631.

25. Lesch MD, Spear JF, Simson MB. A computer model of the electrogram: What causes fractionation? J Electrocardiol.1988;21:S69-S73.

26. Marrouche NF, Wilber D, Hindricks G, et al. Association of atrial tissue fibrosis identified by delayed enhancement MRI and atrial fibrillation catheter ablation: the DECAAF study. JAMA. 2014;311(5):498-506.

27. Oakes RS, Badger TJ, Kholmovski EG, et al. Detection and quantification of left atrial structural remodeling with delayed-enhancement magnetic resonance imaging in patients with atrial fibrillation. Circulation. 2009;119(13):1758-1767.

28. Verma A, Wazni OM, Marrouche NF, et al. Pre-existent left atrial scarring in patients undergoing pulmonary vein antrum isolation: an independent predictor of procedural failure. J Am Coll Cardiol. 2005;45(2):285-292.

29. Yamaguchi T, Tsuchiya T, Nagamoto Y, et al. Long-term results of pulmonary vein antrum isolation in patients with atrial fibrillation: an analysis in regards to substrates and pulmonary vein reconnections. Europace. 2014;16(4):511-520.

30. Kistler PM, Sanders P, Fynn SP, et al. Electrophysiologic and electroanatomic changes in the human atria associated with age. $J \mathrm{Am}$ Coll Cardiol. 2004;44(1):109-116.

31. Providencia R, Lambiase PD, Srinivasan N, et al. Is There Still a Role for CFAE Ablation in Addition to Pulmonary Vein Isolation in Patients with Paroxysmal and Persistent Atrial Fibrillation? A Meta-Analysis of 1,415 Patients. Circ Arrhythm Electrophysiol. 2015;8(5):1017-1029.

32. Sanders P, Morton JB, Davidson NC, et al. Electrical remodeling of the atria in congestive heart failure: electrophysiological and electroanatomic mapping in humans. Circulation. 2003;108(12):1461-1469.

33. Sanders P, Morton JB, Kistler PM, et al. Electrophysiological and electroanatomical characterization of the atria in sinus node disease: Evidence of diffuse atrial remodeling. Circulation. 2004;109(12):1514 1522.

34. Roberts-Thomson KC, Kistler PM, Sanders P, et al. Fractionated atrial electrograms during sinus rhythm: relationship to age, voltage, and conduction velocity. Heart Rhythm. 2009;6(5):587-591.

35. Bao Z, Chen H, Yang B, et al. "Prolonged Sinus Pauses upon Termination of Paroxysmal Atrial Fibrillation: Abnormal Right Atrial Electrophysiologic and Electroanatomic Findings". Texas Heart Institute Journal. 2017;44(2):107-114.

36. Tse G, Liu T, Li KH, et al. Tachycardia-bradycardia syndrome: Electrophysiological mechanisms and future therapeutic approaches. Int J Mol Med. 2017;39(3):519-526.

37. Jackson LR, Rathakrishnan B, Campbell K, et al. "Sinus Node Dysfunction and Atrial Fibrillation: A Reversible Phenomenon?" Pacing Clin Electrophysiol. 2017;40(4):442-450.

38. De Ponti R, Marazzato J, Bagliani G, et al. "Sick Sinus Syndrome". Cardiac Electrophysiol Clin. 2018;10(2):183-195.

39. Dzeshka MS, Lip GY, Snezhitskiy V, et al. Cardiac Fibrosis in Patients With Atrial Fibrillation: Mechanisms and Clinical Implications. J Am CollCardiol. 2015;66(8):943-959. 
40. Wijffels MC, Kirchhof CJ, Dorland R, et al. Atrial fibrillation begets atrial fibrillation. A study in awake chronically instrumented goats. Circulation. 1995;92(7):1954-1968.

41. Everett TH, Li H, Mangrum JM, et al. Electrical, morphological, and ultrastructural remodeling and reverse remodeling in a canine model of chronic atrial fibrillation. Circulation. 2000;102(12):1454-1460.

42. Nattel S, Harada M. Atrial remodeling and atrial fibrillation: recent advances and translational perspectives. $J$ Am CollCardiol. 2014;63(22):2335-2345.

43. Andrade J, Khairy P, Dobrev D, et al. The clinical profile and pathophysiology of atrial fibrillation: relationships among clinical features, epidemiology, and mechanisms. Circ Res. 2014; 114(9):14531468 .

44. Miragoli M, Salvarani N, Rohr S. Myofibroblasts induce ectopic activity in cardiac tissue. Circ Res. 2007;101(8):755-758.

45. Goette A, Juenemann G, Peters B, et al. Determinants and consequences of atrial fibrosis in patients undergoing open heart surgery. Cardiovasc Res. 2002;54(2):390-396.

46. Platonov PG, Mitrofanova LB, Orshanskaya V, et al. Structural abnormalities in atrial walls are associated with presence and persistency of atrial fibrillation but not with age. $\mathrm{J} \mathrm{Am} \mathrm{CollCardiol.}$ 2011; 58(21):2225-2232.

47. Frustaci A, Chimenti C, Bellocci F, et al. Histological substrate of atrial biopsies in patients with lone atrial fibrillation. Circulation. 1997;96(4):1180-1184.

48. Boldt A, Wetzel U, Lauschke J, et al. Fibrosis in left atrial tissue of patients with atrial fibrillation with and without underlying mitral valve disease. Heart. 2004; 90:400-405.

49. Allessie M, Ausma J, Schotten U. Electrical, contractile and structural remodeling during atrial fibrillation. Cardiovasc Res. 2002;54(2):230 246.

50. Li D, Fareh S, Leung TK, et al. Promotion of atrial fibrillation by heart failure in dogs: atrial remodeling of a different sort. Circulation. 1999;100(1):87-95.

51. Nattel S. New ideas about atrial fibrillation 50 years on. Nature. 2002;415(6868):219-226.

52. Röcken C, Peters B, Juenemann G, et al. Atrial amyloidosis: an arrhythmogenic substrate for persistent atrial fibrillation. Circulation. 2002;106(16):2091-2097.

53. Masuda M, Fujita M, Iida O, et al. Left atrial low-voltage areas predict atrial fibrillation recurrence after catheter ablation in patients with paroxysmal atrial fibrillation. Int J Cardiol. 2018;257:97-101.

54. Oakes RS, Badger TJ, Kholmovski EG, et al. Detection and quantification of left atrial structural remodeling with delayed-enhancement magnetic resonance imaging in patients with atrial fibrillation. Circulation. 2009; 119(13):1758-1767.
55. Yamaguchi T, Tsuchiya T, Nakahara S, et al. Efficacy of Left Atrial Voltage-Based Catheter Ablation of Persistent Atrial Fibrillation. $J$ Cardiovasc Electrophysiol. 2016;27(9):1055-1063.

56. Yamaguchi T, Tsuchiya T, Fukui A, et al. Impact of the extent of lowvoltage zone on outcomes after voltage-based catheter ablation for persistent atrial fibrillation. J Cardiol. 2018;72(5):427-433

57. Higuchi K, Cates J, Gardner G, Morris A, Burgon NS, Akoum N, Marrouche NF. The SpatialDistribution of LateGadoliniumEnhancement of Left AtrialMagnetic Resonance Imaging in Patients With Atrial Fibrillation. JACC Clin Electrophysiol. 2018;4(1):49-58.

58. Schreiber D, Rieger A, Moser F, et al. Catheter ablation of atrial fibrillation with box isolation of fibrotic areas: Lessons on fibrosis distribution and extent, clinical characteristics, and their impact on long-term outcome. J Cardiovasc Electrophysiol. 2017;28(9):971-983.

59. Rolf S, Kircher S, Arya A, et al. Tailored atrial substrate modification based on low- voltage areas in catheter ablation of atrial fibrillation. Circ Arrhythm Electrophysiol. 2014;7(5):825-833.

60. Kottkamp H, Berg J, Bender R, et al. Box Isolation of Fibrotic Areas (BIFA): a patient-tailored substrate modification approach for ablation of atrial fibrillation. J Cardiovasc Electrophysiol. 2016;27(1):22-23.

61. Jadidi AS, Lehrmann H, Keyl C, et al. Ablation of persistent atrial fibrillation targeting low-voltage areas with selective activation characteristics. Circ Arrhythm Electrophysiol. 2016;9(3):pii: e002962.

62. Yang G, Yang B, Wei Y, et al. Catheter Ablation of Nonparoxysmal Atrial FibrillationUsing Electrophysiologically Guided Substrate Modification During Sinus RhythmAfter Pulmonary Vein Isolation. Circ Arrhythm Electrophysiol. 2016;9(2):e003382.

63. Nakahara S, Yamaguchi T, Hori Y, et al. Spatial Relation Between Left Atrial Anatomical Contact Areas and Circular Activation in Persistent Atrial Fibrillation. J Cardiovasc Electrophysiol. 2016;27(5):515-523.

64. Cutler MJ, Johnson J, Abozguia K, et al. Impact of voltage mapping to guide whether to perform ablation of the posterior wall in patients with persistent atrial fibrillation. $J$ Cardiovasc Electrophysiol. 2016;27(1):13-21.

65. Yang B, Jiang C, Lin Y, et al. STABLE-SR Investigators*. STABLESR (electrophysiological substrate ablation in the left atrium during sinus rhythm) for the treatment of nonparoxysmal atrial fibrillation: a prospective, multicenter randomized clinical trial. Circ Arrhythm Electrophysiol. 2017;10(11):e05405.

66. Kircher S, Arya A, Altmann D, et al. Individually tailored vs. standardized substrate modification during radiofrequency catheter ablation for atrial fibrillation: a randomized study. Europace. 2018;20(11):1766-1775.

67. Takeshi T. The START Trial (Substrate Targeted Ablation of Persistent Atrial Fibrillation Trial). Johns Hopkins University. 2017. 